\section{LA FLOR DE MI SECRETO (ALMODÓVAR, 1995): LA LITERATURA COMO SEDUCCIÓN}

\author{
Cristina Martínez-Carazo \\ University of California, Davis \\ Department of Spanish \\ One Shields Ave. \\ University of California \\ Davis, CA 95616 \\ Estados Unidos \\ cmmartinezcarazo@ucdavis.edu
}

\begin{abstract}
The multiple references to writing in Pedro Almodovar's La flor de mi secreto (1995) have arguably made this film his most literary. Reading and writing function as the structural axis of the movie because they define the existence of the main character Leo/Amanda Gris. But beyond the constant literary allusions, what makes the "literarity" of this film is, on the one hand, the construction of the protagonist according to literary models and on the other the exploration of her subjectivity through her writing.Leo as a character is created as a replica of the tormented writers that she admires. In their stereotypical tradition, she is an alcoholic, addicted to sleeping pills, suicidal, obsessed with her loneliness and installed in her writing as a survival space. Furthermore, the spectator accesses her subjectivity through her career as a writer because her melodramatic life gets entangled with her novels.Almodóvar projects his literary ghosts in La flor de mi secreto and in the process, subverts the parameters of literature, the canon, the gender and genre categories and the question of authorship. He also resists a stable definition of literature as art by inserting it in a filmic text, favoring a complex intertextuality which contributes to dissolving the frontiers between high and low culture and to opening a common ground for the arts.
\end{abstract}

KEY WORDS: Construction of the character; literarity; subjectivity; gender and genre subversions; intertextuality; high and low culture.

Las múltiples referencias a la escritura presentes en La flor de mi secreto (1995) han llevado a etiquetar esta película como la más literaria de Pedro Almodóvar. Ya en la primera toma de la protagonista, la cámara se desliza en un breve travelín desde su cama hasta una torre de novelas apiladas junto a la mesilla de noche, seguido de una imagen del personaje frente a su máquina de escribir, marcando con

\section{LA FLOR DE MI SECRETO (ALMODÓVAR, 1995): LITERATURE AS SEDUCTION}

RESUMEN: Las múltiples referencias a la escritura presentes en La flor de mi secreto (1995) han llevado a etiquetar esta película como la más literaria de Pedro Almodóvar. La lectura y la escritura funcionan como hilo conductor de la película en la medida en que articulan la existencia de la protagonista, Leo/Amanda Gris. Pero más allá de este despliegue de alusiones literarias, lo que en realidad determina la literaridad del film es, por un lado, la construcción de dicho personaje a partir de modelos literarios y por otro la exploración de su subjetividad a partir de la escritura. Leo se perfila como réplica de las escritoras "atormentadas" a las que admira; alcohólica como ellas, adicta a los tranquilizantes, suicida, abrumada por la soledad e instalada en la escritura como espacio de supervivencia. Además, el espectador accede a su subjetividad a partir de su trayectoria como escritora ya que su melodramática existencia se funde/confunde con su escritura. Almodóvar proyecta en La flor de mi secreto sus fantasmas literarios y subvierte en el proceso los parámetros de la literatura, el valor del canon, las categorias genéricas, tanto literarias como sexuales, las leyes del mercado y las cuestiones de autoría. Opone además una clara resistencia a una definición estable del fenómeno literario al insertarlo en el cinematográfico, activando con ello un complejo juego de intertextualidades que en última instancia contribuyen a disolver las fronteras entre la alta y baja cultura y a abrir un espacio de convergencia entre las artes.

PALABRAS CLAVE: Construcción del personaje; literaridad; subjetividad; subversión sexual y de géneros literarios; intertextualidad; alta cultura y cultura popular.

ello las pautas de texto. Obras de Juan José Millás (Ella imagina), Julio Cortázar (Cuentos completos), Djuna Barnes (El bosque de la noche), Janet Frame (Un ángel en mi mesa), Henri James (La musa trágica) aparecen esparcidas por la habitación mostrando las preferencias literarias de la protagonista. El espectador entra así en contacto con Leo/Amanda Gris, lectora ávida, escritora de novelas rosas 
(y negras), ensayista, declarada admiradora de escritoras "malditas" y víctima de la dinámica del mercado literario (y de un matrimonio fallido). Junto a esta faceta profesional, su problemática vida afectiva se anticipa también por medio de la escritura. Al comienzo de la película vemos a Leo, en un claro estado de desesperación, escribiendo una carta angustiosa a su marido en la que declara: "A veces tu recuerdo [...] me oprime el corazón hasta impedirme respirar" [...] "Te odio. Ojalá pudiera seguir odiándote como te odio ahora". Su fracaso matrimonial y la consiguiente reconstrucción de su vida afectiva vienen igualmente marcados por la escritura en la medida en que su crisis sentimental se refleja en su evolución como novelista y en que su nueva pareja, Ángel, director del suplemento cultural del periódico El País, le abre nuevos caminos en el mundo de la literatura. Vemos así cómo su vida afectiva y profesional se tejen sobre un mismo entramado literario.

El espectador se sitúa frente a una película cuyo germen es la literatura. El propio director al referirse a la construcción del film declara: "The structure resembles that of a novel with chapter headings. I nearly gave a title to each major section of the film: "Leo's Solitude", "The family", "El Pais", "The husband's visit", "The suicide", "The Return to the Village", "The Return to the City". Of course Leo shares each chapter with the other characters. But the narrative is linear because everything relies on her [...] This particular form was demanded by the story. A story always has its own laws" (Strauss, 1996, 162)'. Paul Julian Smith considera esta película como la bisagra que une dos estéticas dominantes en el cine de Almodóvar; la primera, etiquetada por este crítico como "período rosa", correspondiente a sus primeras películas disidentes y excesivas a nivel estilístico y temático, y la última, "período azul" marcada por unas obras más contenidas, menos coloridas, menos provocativas temáticamente. Además, para P. J. Smith, La flor de mi secreto marca la entrada de Almodóvar en el cine de "arte y ensayo" y lo hace en base al cambio de tratamiento que reciben tres de sus temas clave: sexo, ciudad y literatura. En lo que atañe a la cuestión sexual, La flor... reemplaza los personajes sexualmente atípicos de sus primeras películas por parejas heterosexuales convencionales; el tratamiento de la ciudad pierde su perfil delirante para mostrarse como un espacio problemático y alienante, y respecto a lo literario, el lenguaje espontáneo e improvisado de su primera fase es sustituido por diálogos y referencias intencionalmente literarios: "An early spontaneity, even improvisation, of narrative and dialogue gives way to a much more selfconscious literarity and appeal to texts as precedents" (Smith, 2003, 150).

Más allá de este despliegue de alusiones literarias, lo que en realidad determina la literaridad del film es, por un lado, la construcción de la protagonista a partir de modelos literarios y, por otro, la articulación de su existencia a partir de la escritura y en esta doble función de lo literario centraré mi estudio. Con ello pretendo mostrar cómo al margen de las alusiones obvias a la literatura y de la omnipresencia de la palabra escrita en el film, Almodóvar hace de la literatura la esencia de su film, y cómo a su vez la literatura le permite cuestionar las categorías genéricas (sexuales y literarias), insertando esta obra, en apariencia menos provocadora y más contenida que las anteriores, en la corriente desestabilizadora que atraviesa toda su producción cinematográfica.

En primer lugar, el personaje de Leo se construye a imagen y semejanza de las autoras que ella lee y admira. El director penetra en la subjetividad del personaje por medio de la literatura, permitiendo que ella se desvele a sí misma a través de su escritura y de sus lecturas. Con ello crea en el espectador un efecto en apariencia contradictorio respecto al personaje, distanciador por cuanto su dimensión de escritora la dota de un halo ficticio, y aproximador, en cuanto a que la escritura permite una exploración de su subjetividad que, de otro modo, no lograría esta profundidad. Esta dialéctica entre proximidad y distancia, lejos de dañar la conexión entre la audiencia y el film, estrecha el vínculo entre ambos al entregar al espectador un personaje complejo y creible, abrumado por una problemática sentimental claramente reconocible.

El peso del componente literario se manifiesta en primer lugar en el modo en que Leo se perfila a sí misma como réplica de las escritoras "atormentadas" a las que admira, insinuando que no es sólo la producción novelesca de estas mujeres lo que la atrae, sino su persona, su vida marginal y desgarrada, su condición de alcohólicas, su adicción a los tranquilizantes, sus inclinaciones suicidas, su desgarrada soledad $y$, sobre todo, su instalación en la escritura como espacio de supervivencia. La trayectoria vital de Leo viene así jalonada por la conducta de sus escritoras modelo; inclinada al alcohol y a los fármacos como ellas, suicida, 
atormentada; en una palabra, infeliz. Djuna Barnes, Virginia Wolf, Dorothy Parker, Edith Warthon, Janet Frame funcionan como musas vitales más que literarias. En ellas se reconoce Leo como víctima de sí misma y hace suyo su fracaso. En el contexto melodramático del film, las escritoras son un modelo de infelicidad para la protagonista, un molde para configurar su propia desgracia. La literatura sirve para marcarle al espectador el itinerario emocional y las pautas de la desesperación de la protagonista, que al hacer suya una frase de Djuna Barnes -"Tienes enfrente de ti a una mujer creada para la ansiedad"- parece estar mostrándole al público el guión de su historia.

Para que este proceso de identificación con las escritoras mencionadas mantenga coherencia, Leo se ciñe al tipo de literatura que sus modelos producen. De ahí que opte por sustituir el género que le ha llevado a la fama, la novela rosa, por una escritura susceptible de reflejar su caótica interioridad. Vivir atormentada y dedicarse a la literatura rosa resulta a todas luces inverosímil. A ello alude el propio Almodóvar al afirmar: "Si una escritora de novela rosa, por despiste o por dolor, se contempla en el negro espejo de la realidad, equivocó su mirada (la mirada del escritor es esencial) y habrá cruzado la frontera que divide dos géneros tan opuestos y vecinos como el rosa y el negro" (http:// www.clubcultura.com/clubcine/clubcineastas/almodovar).

Además de este cruce de colores literarios al que volveremos más adelante, lo que verdaderamente marca el protagonismo de la literatura en el film es la articulación de la subjetividad del personaje por medio de la escritura. Su trayectoria vital viene marcada a golpe de teclado. De la novela rosa que consolida su carrera literaria, réplica de sus años de éxito, a la escritura de novelas negras, eco de su tormentosa relación matrimonial, su vida y su literatura se funden/confunden. Leo se presenta en la película como una mujer que ha triunfado social y profesionalmente gracias a la literatura. Su origen humilde, inferido a partir de la baja posición social de su madre y de su hermana, ha quedado atrás gracias a las sustanciosas ventas de sus novelas rosas, firmadas bajo el pseudónimo de Amanda Gris. La mujer culta y sofisticada que emerge de este éxito profesional y que ejemplifica en su persona la temática rosa, se ve abatida por una gran crisis sentimental, debida al distanciamiento físico y emocional del marido y como consecuencia cambia de estilo ${ }^{2}$. A partir de esta crisis Leo escribe y se inscribe a sí misma como personaje de novela negra. Su abatimiento le impide seguir las normas de su contrato editorial -entregar cinco "novelas de amor y de lujo" al año durante los próximos tres años, situadas en "escenarios cosmopolitas, sol radiante, urbanizaciones, con subsecretarios, ministros, yuppies, nada de política, ausencia de conciencia social, hijos ilegítimos, final feliz"- y la lleva a reemplazar la artificiosidad de la novela rosa por el realismo de la novela negra. De ahí que su editora se indigne ante este giro literario de Amanda Gris y le recuerde con acritud que la literatura es un medio de evasión que no deja espacio para lo real: "cuando alguien compra una novela nuestra quiere olvidarse de la sordidez en la que vive [...] ¡La realidad! Bastante realidad tenemos en nuestra casa! [...] ¡La realidad debería estar prohibida! [...] Las novelas sirven para recuperar la ilusión de vivir". Le reprocha además que la editorial haya cumplido a rajatabla sus condiciones como escritora: no mostrar su nombre, ni su fotografía, y no conceder entrevistas para mantenerla en el más absoluto anonimato y que ella sea incapaz de ajustarse al contrato. Por si la cuestión legal no fuera suficiente, le recuerda displicentemente los beneficios económicos que las novelas rosas le han reportado: "Piensa en tu chalet, tus viajes al Caribe, tus liposucciones, colágeno, tu familia pobre...".

Será su rebelión y su reinvención a partir de la lectura y de la escritura -lectora de escritoras atormentadas, autora de novelas rosas y negras y de ensayos literarios- la que posibilite la reformulación de su existencia. A esta cuestión alude Alejandro Yarza al afirmar que: "El sujeto que emerge del cine de Almodóvar es, como veremos, un sujeto-máscara que representa un papel social, producido por la intersección de una serie de discursos y prácticas sociales que lo construyen como tal. No es entonces un sujeto trascendental y unitario que precede al discurso sino uno múltiple y contradictorio que se origina en éste" (Yarza, 1999, 34). Este sujeto cambiante, producto de los discursos que jalonan su existencia, encuentra su réplica en su producción literaria. El primer paso en su proceso de transformación viene dado por la escritura de una novela negra, La cámara frigorífica ${ }^{3}$, una delirante y truculenta historia. Recordemos que este manuscrito pasa por un sinfín de avatares: es rechazado por la editorial, robado por el hijo de su asistenta, Antonio, y vendido a Bigas Luna para convertirlo en guión de cine, posibilitando con ello la financiación de un espectáculo de flamenco fusión protagonizado por Antonio (Joaquín

ARBOR Vol. 187748 marzo-abril [2011] 383-390 ISSN: 0210-1963

政


Cortés) y por su madre, Blanca (Manuela Vargas). El fracaso editorial del manuscrito da paso a la nueva Leo, autora ahora de ensayos literarios y ligada emocionalmente a otro escritor/editor, Ángel. Pero para ello habrá de pasar por un doble suicidio, literario y real. El primero, metafórico, se consuma por medio de la escritura de un ensayo destructivo sobre su propia literatura rosa, publicado en El País también bajo pseudónimo. Leo, que gracias a su amiga Betty entra en contacto con Ángel, le entrega en su primer encuentro el manuscrito de su última novela, La cámara frigorífica, y dos ensayos ${ }^{4}$. Después de leer estos textos y fascinado por su estilo, Ángel concierta una segunda cita para concretar su colaboración en el periódico. Leo aclara desde el primer momento que prefiere escribir sólo sobre lo que le gusta y evitar la literatura española a ser posible. Ángel, inesperadamente, le pregunta qué le parece Amanda Gris sin saber que ella se oculta tras este seudónimo. Indignada responde: "Me horroriza Amanda Gris [pero] no quiero escribir en contra de ella. Ya hay suficientes cosas negativas en mi vida". Y añade que este género le repugna ya que "no hay dolor, solo rutina, complacencia y sensiblería". Ángel aprovecha su consternación para pedirle que desarrolle en cinco folios lo que le ha dicho y de ahi sale su artículo "suicida", una ácida autocrítica de sus novelas rosas, titulada "¿Es Amanda Gris una buena mecanógrafa?"5, firmado bajo el seudónimo Paz Sufrategui ${ }^{6}$. La respuesta de Ángel no se hace esperar y publica inmediatamente en su periódico el artículo de Leo junto con otro suyo en su defensa titulado "Amanda Gris, la Alejandro Dumas de la novela sentimental". Pero la pasión de Ángel por el género rosa no acaba en este elogio sino que se prolonga en la escritura de tres novelas en las que suplanta a Leo, solucionando así la falta la inspiración de la escritora y librándola con ello de las ataduras mercantiles de su contrato con la editorial. Vemos con ésto, cómo por medio de la escritura Leo, cancela su pasado como autora de novelas rosas y abre una nueva etapa dedicada a la crítica literaria. El segundo suicidio, éste físico, inducido por el alejamiento de su marido, se concreta por medio de la ingestión de un tubo de tranquilizantes. Será la voz de su madre en el contestador del teléfono, la que la saque de su letargo, le haga vomitar las pastillas y la devuelva a la vida. De ambos suicidios emergerá la nueva Leo Macías, despojada de su máscara de Amanda Gris, colaboradora ahora del suplemento literario de El País y vinculada sentimentalmente a su editor.
La literatura pasa así a funcionar como eje en la articulación de la subjetividad de los personajes y del texto filmico. Los textos literarios que Leo y Ángel producen y leen dibujan el mapa de su existencia. En el caso de Leo el sistema simbólico sobre el que ella se constituye como sujeto no es sólo el lenguaje, sino el lenguaje literario, que opera como catalizador de su realidad. La cadena de experiencias que engarzan su trayectoria vital (éxito profesional, fracaso matrimonial, soledad, dependencias...) sumada a su posición como lectora y escritora, cobra consistencia al transformarse en literatura. Se establece así un juego de influencias mutuas en el que los modelos literarios marcan las pautas de su existencia a la vez que sus avatares personales se metamorfosean en literatura. El deseo como móvil de la existencia aparece suplantado por el deseo de la representación: Leo representa (es decir, ejemplifica y narra) la postura existencial de los personajes de las novelas rosas que escribe en su primera fase literaria (mujer enamorada, social y profesionalmente exitosa) y la de los personajes de la novela negra de su segunda etapa de escritora (seres atormentados por su abrumadora existencia, infelices). Igualmente, su enajenación a raíz del fracaso matrimonial y su incapacidad para seguir escribiendo literatura rosa, se proyecta claramente en las novelas negras de su segunda época, en concreto en La cámara frigorifica, al igual que en los temas que elige para sus ensayos, las escritoras infelices y atormentadas a las que tanto admira y la destrucción literaria de Amanda Gris.

Pero a pesar de esta coherencia emocional entre la escritora y su obra, Leo se presenta como un personaje plagado de contradicciones como lectora y como escritora. Sus sofisticadas preferencias literarias a juzgar por sus lecturas contrastan con la artificialidad y sensiblería de sus novelas rosas y con lo pedestre de la novela que acaba de escribir, La cámara frigorífica. A ello se suma su ambigua postura respecto al manuscrito de dicha novela (por un lado se proyecta en él y por otro lo califica de basura, lo envía publicar y se lo rechazan pero a la vez no lo registra porque no pensaba publicarlo, se muestra indiferente ante el robo del texto y su futura vida como guión cinematográfico de Bigas Luna; finalmente se alegra de que haya servido para dar vida al espectáculo de flamenco de Antonio). Si bien esta conducta le hace perder consistencia como escritora le lleva a ganarla como personaje libresco ${ }^{7}$. 
Otra función clave que desempeña la literatura en la película es revelar la insatisfacción de los escritores con su persona literaria poniendo con ello en tela de juicio la libertad del artista y desvelando la tiranía del mercado. No es sólo Leo la afectada por el deseo de producir otro tipo de textos sino que Ángel padece el mismo mal. Uno de los sintomas de esta insatisfacción profesional es ocultarse bajo diversos seudónimos manteniendo asi en secreto su verdadera identidad. Leo firma sus novelas rosas con el nombre de Amanda Gris y sus ensayos críticos con el de Paz Sufrategui. Su desprecio por el género rosa, patente en los calificativos que le atribuye (falso, sensiblero, complaciente) por un lado, y el contenido auto-crítico de su ensayo, justifican estas ocultaciones. Ya al referirse a Amanda Gris, declara que la única cualidad que reconoce en esta escritora es la de ocultarse bajo un pseudónimo. A su vez, Ángel, que como editor del suplemento literario de El País está obligado a escribir ensayos y a respetar el canon, oculta su pasión por la literatura rosa y publica su artículo a favor de Amanda Gris bajo el seudónimo de Paqui Derma. El juego de ocultaciones conlleva además una serie de connotaciones vinculadas a la cuestión del género sexual. Ángel se muestra fascinado por la novela rosa, netamente femenina y adscrita a la cultura popular; de ahí su destreza al suplantar a Leo en la escritura de las dos novelas que le exige el contrato editorial. Tal es su dominio del género que la editora llega a afirmar que las suyas son las dos mejores obras de Amanda Gris. Por otro lado, con total congruencia literaria, al referirse a las novelas rosas las considera un género típicamente femenino, lo cual explica su elección de un nombre femenino, Paqui Derma, para firmar el artículo en defensa de Amanda Gris. A esto se suma el hecho de hablar de sí mismo en femenino. Al preguntarle Leo quién es Paqui Derma él contesta, "servidora" y añade, "es que estoy un poquito foca", ampliando con ello el sentido de su nombre ficticio. Almodóvar pone así en tela de juicio la validez del canon y la integridad de la crítica literaria, doblemente amenazada por el hecho de que Ángel, además de suplantar a Leo, declara haber hecho de negro de otros escritores ${ }^{8}$.

La escritura lleva así a desestabilizar las cuestiones genéricas tanto a nivel literario como sexual, primero por las insólitas inclinaciones literarias de quienes definen el canon -los críticos- y segundo por las repetidas suplantaciones de Ángel. La literatura rosa, asociada a lo femenino, se adscribe a Ángel y la negra, asociada a lo masculino, a
Leo. Como ha estudiado José Colmeiro: "La semiótica del color fija una serie de códigos cromáticos establecidos y culturalmente ligados a concepciones del género sexual y literario que Almodóvar aprovecha y subvierte en su cine, reclamando la libertad de resignificar estos códigos de acuerdo a unos valores contrarios que desestabilizan el sistema de representación simbólico tradicional" (Colmeiro, 1997, 117). El mismo Almodóvar alude a la simbología de los colores para explicar la trayectoria vital y profesional de la protagonista. En estos términos plasma su visión: "El cambio que se opera en la vida de Leo es un cambio de color, casi un trasvase de colores entre vida y escritura. Al principio escribe novela rosa pero su vida es muy negra. Al final sucede lo contrario, sus perspectivas de vida son mucho más cálidas (no exactamente rosas, eso sería irreal) pero sí anaranjadas, como el cielo al atardecer, o el fuego de una chimenea. Respecto a su escritura, aunque no se especifique en la película, yo puedo adelantar que será negra y esperpéntica, inspirada en la realidad que llena las páginas de sucesos de los periódicos. Leo tiene varias carpetas, llenas de recortes de periódicos, con sucesos extraordinarios. En La flor casi no se ven, pero están sobre su mesa de trabajo" (http://www.clubcultura.com/clubcine/clubcineastas/almodovar).

Pero al margen de sus diferencias, estos dos modos de escritura convergen en su contenido melodramático. Tanto el sentido etimológico del término melodrama como el popular, impregnan el texto fílmico y los textos literarios incrustados en él. Por un lado, la música inherente al melodrama acompaña y matiza la trayectoria emocional del personaje. La canción de Chavela Vargas "El último trago" duplica la crisis matrimonial de Leo ("quiero ver a qué sabe tu olvido") y anticipa su relación con Ángel ("Otra vez a brindar con extraños") ${ }^{9}$. Si bien la esencia del bolero radica en el dolor, el abandono y la soledad, en este caso abre paso también a la superación de este deplorable estado de ánimo. El dolor que exhibe el yo poético en esta canción y su modo de ahogar las penas en alcohol se hacen eco del sentir de la protagonista, expandiendo y verbalizando su estado de ánimo. Esta universalización de las penas de amor inherente al bolero corrobora la afirmación de Monsivais al etiquetarlo como: "the autobiography of everyone and no one" (192). Igualmente el tema de Bola de Nieve "Ay, amor" amplía la desolación de Leo que, tras desmayarse en los brazos de Ángel, despierta desorientada en la cama de éste con la canción del cantante cubano como 
fondo. "Si sólo queda en mí dolor y vida, jay, amor! No me dejes vivir". Este "dolor y vida" de la canción dará además título al artículo de Leo sobre las escritoras atormentadas y aparecerá también, accidental o intencionadamente, en un "post-it" sobre el manuscrito de La cámara frigorífica que Leo tira a la papelera y Antonio recupera. Con ello, este tema, "Dolor y vida", funciona como leitmotiv de la obra y marca el tono emocional del film. El registro en el que se mueve el bolero -pasiones desgarradoras, amores imposibles, abandono, soledad, dolor- se corresponde, como se ha visto, con el de la protagonista, pero Almodóvar con su enorme capacidad desestabilizadora, complica esta lectura unívoca y utiliza el bolero, además como punto de partida para superar una crisis emocional. A ello se refiere Kathleen Vernon al señalar que: "Paradójicamente, en $L a$ flor la capacidad del bolero para expresar el sentimiento trágico de la vida se vincula con su poder regenerativo" (Vernon, 2007, 253).

En su afección popular y referido al cine, el término melodrama se aplica por un lado a películas con un alto contenido emocional y con una voluntad realista que aspira a legitimizar el exceso emotivo $y$, por otro, se adscribe a obras que inducen la emoción con un sentimentalismo exagerado más que con el contenido mismo de la historia, cargándose aquí de connotaciones negativas. La película que nos ocupa exhibe una sucesión de melodramas dentro del melodrama anticipados ya en el título auto-confesional y melodramático - La flor de mi secreto- y en el cartel anunciador, un corazón hecho de rosas de color rosa con la silueta protagonista en negro incrustada en él, imagen que además juega con los géneros literarios que cultiva Leo, rosa y negro. La misma carga melodramática domina la primera secuencia del film, correspondiente a la grabación en video de una simulación sobre la donación de órganos, en la que un par de médicos intentan convencer a una madre abrumada por el dolor para que done los órganos de su hijo muerto en un accidente ${ }^{10}$. Asociada a la simulación, aparece una lista de sentimientos (pena, rabia, dolor, impotencia, alivio, soledad, vacío, frustración, injusticia) que serán los mismos que floten en la vida de Leo y en los numerosos textos e historias que convergen en su persona: la situación familiar de su hermana y su madre, su profesión de escritora de novelas rosas y negras, su propia existencia melodramática, el bolero de Chavela Vargas, el filin de Bola de Nieve, el show flamenco de Antonio y Blanca, "Soledad".
Pero donde el melodrama se tematiza y se cuestiona es en la escritura de Leo. La novela rosa y la novela negra que marcan su trayectoria literaria condensan esta esencia melodramática. El propio Almodóvar alude directamente a su modo de entender los trasvases entre estos dos géneros. Para él: "Aunque mantengan cierto parentesco no deben confundirse la novela rosa con el melodrama. Toda novela rosa es melodramática, pero no todo melodrama es rosa, en absoluto" (Almodóvar http://www.clubcultura. com/clubcine/clubcineastas/almodovar).

Esta diferenciación de géneros literarios se densifica al insertarla en el contexto melodramático del film y cobra especial sentido al explorar la dialéctica entre la literatura y la subjetividad del personaje. Almodóvar recurre al melodrama para sacar a la luz las tensiones emocionales que operan en la vida de la protagonista y para plasmar la intersección entre su literatura, novela rosa y novela negra, y su propia existencia. Este cambio de tono en la novelistica de Leo viene mediado por la búsqueda de autenticidad, incompatible en su opinión con el género rosa. Su incapacidad para seguir escribiendo en este registro, a pesar de lo lucrativo que le resulta, emana de la falsedad que encierra, pues en su opinión "No se ocupa en lo más mínimo de los sentimientos".

Como bien muestra el film, Leo no se acerca a la literatura movida por el valor intrínseco de los textos sino por los sentimientos que evocan, unos sentimientos que afloran igual a partir de un bolero que de un libro y que la separan de toda postura intelectual. Se siente inevitablemente imantada por obras que ahondan en estados de ánimo similares al suyo y le permiten verse reflejada en ellos. Desasida emocionalmente debido a su fracaso amoroso va en busca de modelos que legitimen su dañada afectividad, que le ayuden a verbalizarla y a mantener la cordura, en medio de ese miedo a la locura que, como un mal aire, sopla sobre toda su familia. No es una escritora que se acerca a la literatura desde una perspectiva intelectual sino una mujer desbordada por su dolor que busca modelos en los que reconocerse; una mujer perdida "como vaca sin cencerro" como bien le recrimina su madre, que trata de encontrarse en el dolor literario de otros. A partir de aquí la ecuación autenticidad, novela negra y realidad funciona como matriz de la nueva Amanda Gris"1. De ahí que rechace la novela rosa porque su sustrato emocional es diametralmente opuesto al suyo y se reconozca en la 
"novela negra y esperpéntica". A esta convergencia entre escritor y escritura alude M. Allinson al afirmar que: "It is one of Almodóvar quirks that a melodramatic story teller should be framed in a melodrama of her own life. As if to reconfirm the melodrama within melodrama" (Allinson, 2001, 142).

Pero al margen de este cóctel de emociones excesivas ${ }^{12}$, Almodóvar declara que la película no es un melodrama, sino un drama sobre el dolor (Strauss, 1996, 164) y que contuvo al máximo la puesta en escena para evitar lo melodramático. Esto explica la tensión entre el melodrama y el esfuerzo por contenerlo que atraviesa la película. No obstante la contención en La flor de mi secreto sólo se entiende dentro del contexto hiperbólico que caracteriza el cine de Almodóvar. Si bien es verdad que el film está más anclado en el realismo y que ha limado muchos de los excesos de su producción anterior, es innegable que Almodóvar como artista se mantiene fiel a sus parámetros estéticos.

Refiriéndose a la literatura, Almodóvar afirma que su capacidad para calar en los sentimientos humanos es superior a la del cine lo cual justifica que en esta película, que para él gira en torno a los sentimientos, escoja la literatura como eje articulador y trace a partir de ella el mapa emocional de la protagonista. A su vez, la singular concepción de la literatura que se proyecta en esta película subvierte en el proceso los parámetros de la palabra escrita, el valor del canon, las categorías genéricas, las leyes del mercado y las cuestiones de autoría. El valor de la obra literaria como producto cultural ya no radica en su calidad intrínseca, sino en factores tan aleatorios como el éxito de ventas, la capacidad producir/reproducir sentimientos auténticos, la fluidez de los géneros. En esta voluntad desestabilizadora encaja la lectura del film de Annabel Martín en la que afirma que La flor de mi secreto junto con ¿Qué he hecho yo para merecer esto? y Tacones lejanos "muestran cómo la producción de conocimiento, una área reservada a la racionalidad y al elitismo masculinizante de la alta cultura, surge también de la experiencia emotiva de la lágrima y el sentir" (Martín, 1997, 16).

En una reflexión sobre las artes Almodóvar declara: "Es cierto que todos los géneros, literarios y cinematográficos alternan entre sí. Se influyen, dividen y subdividen y se mezclan cada vez más. Esta promiscuidad general y generosa de los géneros (eclecticismo, mestizaje) es característica de un siglo generoso que hace balance antes de pasar a la historia" (http://clubcultura.com/clubcine/ clubcineastas/almodovar/esp).

Desde esta visión desestabilizadora propia de la postmodernidad se entiende la articulación de la subjetividad de la protagonista de La flor de mi secreto, personaje cinematográfico y libresco, perfilado a partir de modelos literarios, que se muestra ante el espectador por medio de su compleja relación con la literatura. Esta construcción opone una clara resistencia a la separación de lenguajes artísticos amalgamando en la película el discurso literario y el fílmico. La intertextualidad y la interdisciplinaridad dan así otra vuelta de tuerca al trascender su sentido primero como diálogo entre diferentes textos y al fundir lo verbal y lo visual en una misma obra. El cine de Almodóvar se resiste a la ley unificadora de los géneros y estilos, y abre un espacio de convergencia entre las artes en el que se anulan las categorias ideológicas y estéticas tradicionales.

\section{NOTAS}

1 Refiriéndose al componente literario en esta película Antoine Jaime apunta: "El esmero excesivo que dedica a la escritura es un reproche que se le hizo a Almodóvar a propósito de La flor de mi secreto; los diálogos de esta película, efectivamente, han sido considerados demasiado literarios" (Jaime, 2000, 297).

2 Marsha Kinder subraya la convergencia entre Almodóvar y Buñuel en el tratamiento extremo del amor en sus films. "Yet both use the scandalous excesses of l'amour fou to possition these conceptual and emotional challenges within the familiar genre
Aceptado: 31 de marzo de 2009

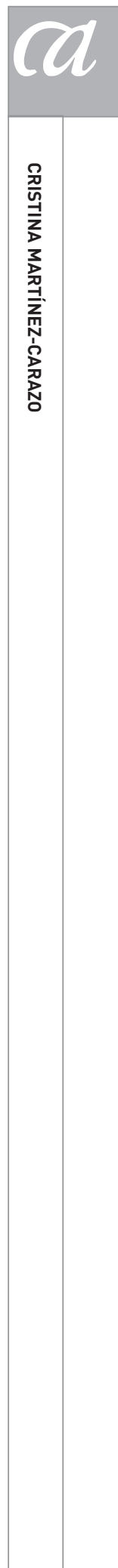


of melodrama, whose hybridity and tonal range make it capable of accommodating such extremes" (Kinder, 2009).

3 Como es bien sabido, este manuscrito será el embrión de la película Volver (Almodóvar, 2006).

4 Recordemos que, además de ser amiga de Leo, Betty es amante de su marido.

5 Un ejemplo más de la omnipresencia de la literatura en esta película es la alusión a Truman Capote que, en un comentario misógino, propone distinguir entre una escritora y una mecanógrafa.

6 Nombre de pila de la responsable de prensa de la productora de Pedro y Agustín Almodóvar, El Deseo.

7 A pesar de estas inconsistencias Leo nunca pierde coherencia como personaje. Edward Guthman señala este aspecto al afirmar que: "Almodóvar is such a great writer and director that he can respect and sympathize with Leo and simultaneously moch her without loosing credibility" (Guthmann, 1996, 44).

8 Javier Escudero considera además que el uso de un seudónimo femenino por parte de Ángel revela la hipocresía de quienes definen el canon literario.

9 Para un estudio completo de la música en la obra de Pedro Almodóvar ver el ensayo de Kathleen Vernon titulado "Las canciones de Almodóvar".

10 R. Prout efectúa una lectura de esta secuencia de la simulación vinculándola con el proceso de redefinición de la identidad nacional en la España actual.
11 A esta cuestión se refiere Margarita Pillado al afirmar que "Lo que justifica la intersección de la novela rosa, el melodrama y la telenovela en La flor de mi secreto es la doble articulación melodramático realista que caracteriza estos tres géneros" (Pillado, 1996, 328).

12 M. D'Lugo explora el contenido melodramatico del film y considera que la secuencia en que se simula la donación de órganos "introduces the concept of emotional excess in melodrama as artifice to be modulated for effect" (D'Lugo, 2006, 88).

\section{BIBLIOGRAFÍA}

Allinson, Mark (2001): A Spanish Labyrinth, London\&New York, IB Tauris.

Almodóvar, Pedro. http://www.clubcultura. com/clubcine/clubcineastas/almodovar

Cabello Castellet, George y Jaume Martí Olivella (eds.) (2000): Cine-Lit, CorvaIlis, OR, Oregon State University.

Colmeiro, José (1997): "Del rosa al negro: subtextos culturales en La flor de mi secreto", Arizona Journal of Hispanic Cultural Studies 1, pp. 115-128.

D'Lugo, Marvin (2007): "El extraño viaje alrededor del cine de Almodóvar", en Javier Herrera y Cristina MartínezCarazo (eds.), pp. 199-218.

- (2006): Pedro Almodóvar, Urbana and Chicago, University of Illinois Press.

Escudero, Javier (2000): "La flor de mi secreto de Pedro Almodóvar: entre casticismo y regeneración", en George Cabello Castellet y Jaume Martí Olivella (eds.), pp. 137-143.
Guthmann, Edward (1996): "Review: The Flower of my Secret", San Francisco Chronical, March 22nd.

Herrera, Javier y Cristina Martínez-Carazo (eds.) (2007): Hispanismo y cine, Madrid, Iberoamericana.

Jaime, Antoine (2000): Literatura y cine en España, Madrid, Cátedra.

Kinder, Marsha (2009): "Mad love and melodrama in the films of Buñuel \& Almodóvar" (En prensa en Letras Peninsulares).

Martín, Annabel (1997): "Almodóvar se tiñe de rosa: reinscripciones melodramáticas del conocimiento y del sentir", en George Cabello Castellet y Jaume Martí Olivella (eds.), pp. 14-22.

Monsivais, Carlos (1997): Mexican Postcards, London, Verso, pp. 166-195.

Pillado, Margarita (1996): "Entre el capricho y la obligación: el efecto de realidad en La flor de mi secreto, de Pedro Almodóvar", Moenia, 2, pp. 327-336.

Prout, Ryan (2004): "All about Spain: transplant and identity in La flor de mi secreto and Todo sobre mi madre", Studies in Hispanic Cinemas 1:1, pp. 43-62.

Smith, Paul Julian (2003): Contemporary Spanish Culture, Cambridge, UK, Polity Press.

Strauss, Frédéric (ed.) (1996): Almodóvar on Almodóvar, London \& Boston, Faber and Faber.

Vernon, Kathleen (2007): "Las canciones de Almodóvar", en Javier Herrera y Cristina Martínez-Carazo (eds.), pp. 241256.

Yarza, Alejandro (1999): Un caníbal en Madrid, Madrid, Libertarias. 